\title{
Prevalencia de acontecimientos potencialmente traumáticos en universitarios españoles
}

\author{
Noemí Pereda*, Maria Forns, y Judit Abad
}

Grup de Recerca en Victimització Infantil i Adolescent (GReVIA). Universitat de Barcelona (Barcelona, España)

\begin{abstract}
Resumen: Objetivos: La prevalencia de acontecimientos potencialmente traumáticos en todas las sociedades y contextos es más alta de lo que podría suponerse. El objetivo de este trabajo es describir aquellos acontecimientos potencialmente traumáticos experimentados por una muestra de jóvenes universitarios españoles. Métodos: Se aplicó el Traumatic Life Events Questionnaire a 1033 estudiantes universitarios $(M=21.71$ años; $D T=$ 2.63) de la Universidad de Barcelona. Resultados: Un 92.3\% de los estudiantes (93.4\% de los varones y $91.8 \%$ de las mujeres) manifestaron haber experimentado al menos un acontecimiento potencialmente traumático a lo largo de su vida. La media de acontecimientos distintos vivenciados fue de $3.58(D T=2.47)$. Se observaron diferencias significativas en el número de acontecimientos experimentados por varones $(M=3.86 ; D T=2.60)$ y mujeres $(M=3.45 ; D T=2.40)$. Conclusiones: La prevalencia de acontecimientos potencialmente traumáticos en población universitaria española es más elevada que la encontrada en estudios publicados previamente. Las diferencias en los acontecimientos que experimentan ambos sexos deberían tenerse en cuenta al evaluar e intervenir en este tipo de situaciones. La importancia de la valoración personal del encuestado respecto a la vivencia de acontecimientos potencialmente traumáticos debe considerarse.
\end{abstract}

Palabras clave: Prevalencia; trauma; universitarios; España.

\section{Introducción}

La violencia interpersonal ha sido considerada uno de los principales problemas de salud pública que tienen que afrontar las sociedades de todo el mundo (OPS, 2002). En este sentido, la violencia se constituye en un acontecimiento potencialmente traumático para el individuo y generador, en muchos casos, de un importante malestar que conlleva consecuencias adversas para la salud. A estas experiencias de violencia se añade la vivencia de otro tipo de acontecimientos, de carácter no intencional, pero que también pueden ser generadores de trauma y tener serios efectos en el bienestar psicológico de los individuos, que podríamos catalogar como potencialmente traumáticos.

La definición de acontecimiento traumático utilizada en la actualidad surge como suceso precipitante del trastorno por estrés postraumático y es descrito, originariamente en el DSM-III, como "un acontecimiento psicológicamente desagradable, que se encuentra fuera del marco normal de la experiencia habitual" (DSM-III; American Psychiatric Association [APA], 1980). El DSM-IV-TR (APA, 2002) define el acontecimiento traumático como aquél en que la persona ha experimentado, presenciado o le han explicado uno (o más) acontecimientos caracterizados por muertes o amenazas para su integridad física o la de los demás (Criterio A1) y la persona ha respondido con un temor, una desesperanza o un horror intensos (Criterio A2). Por tanto, la descripción de este tipo de acontecimientos ha ido ampliándose progresiva-

* Dirección para correspondencia [Correspondence address]: Noemí Pereda. Departament de Personalitat, Avaluació i Tractament Psicològic. Facultat de Psicologia. Passeig Vall d'Hebron 171. 08035 Barcelona (España). E-mail: npereda@ub.edu
Title: Prevalence of potentially traumatic events in Spanish university students.

Abstract: Aims: The high prevalence of traumatic events among different populations has been shown in several studies. The aim of the present study is to establish the prevalence of potentially traumatic events among Spanish university students. Methods: The sample comprised 1033 undergraduates $(M=21.71$ years; $S D=2.63)$. Data were obtained using the Traumatic Life Events Questionnaire (TLEQ) (Kubany y Haynes, 2001). Results: Ninety-two per cent of subjects ( $93.4 \%$ males and $91.8 \%$ females) had been exposed to at least one potentially traumatic event. Males experienced a greater mean number of events than females (males $=3.86, S D=$ 2.60 ; females $=3.45, S D=2.40)$. Sex differences were also found in the appraisal of events as being traumatic and causing major distress. Conclusions: Frequency of exposure to potentially traumatic events was higher in this sample of university students than in previous studies with similar samples. The results suggest the need for trauma screening and intervention, taking into consideration the substantial gender variation. Also the individual's appraisal for considering an event traumatic underlines the importance of taking the individual's perception into account. Key-words: Prevalence; trauma; university students; Spain.

mente $y$, de la exigencia de que el suceso debería encontrarse fuera de la experiencia humana habitual, se pasa en la actualidad a incluir sucesos que no necesariamente revisten esta característica de anormalidad y que no tienen porqué haber sido experimentados por el individuo propiamente pero que deben producir reacciones emocionales intensas, conformando las denominadas víctimas indirectas y latentes (Sigales, 2006). Estas especificaciones han conducido a una ampliación de los sucesos potencialmente traumáticos y, por tanto, a una ampliación de los colectivos vulnerables a desarrollar sintomatología postraumática (Báguena, 2001).

Cabe señalar que este tipo de sucesos no ocurren únicamente en segmentos marginales de la población sino que se encuentran presentes en todas las sociedades y contextos (McCall y Resick, 2003), por lo que se hace necesario analizar el volumen de población afectada por este tipo de experiencias. Los estudios llevados a cabo indican que su prevalencia es más alta de lo que podría suponerse, con porcentajes en población general que superan la mitad de los encuestados (Flett, Kazantzis, Long, MacDonald y Millar, 2002; Kessler, Sonnega, Bromet, Hughes y Nelson, 1995) y se acercan, en la mayoría de estudios norteamericanos a prácticamente su totalidad -77\%, 81\% y 90\%- (Breslau et al., 1998; Robin, Chester, Rasmussen, Jarason y Goldman, 1997; Stein, Walker, Hazen y Forde, 1997), si bien los estudios epidemiológicos europeos han obtenido cifras ligeramente inferiores (DarvesBornoz et al., 2008).

La elevada prevalencia de acontecimientos potencialmente traumáticos encontrada en trabajos llevados a cabo con estudiantes universitarios muestra que este colectivo no parece estar protegido de este tipo de experiencias (Vrana y Lauterbach, 1994). Si bien las muestras de universitarios se han considerado de conveniencia, predominantemente formadas 
por jóvenes de clase media y nivel educativo familiar alto, su uso ha sido defendido al formar un grupo que no difiere en muchas áreas de los resultados que se obtienen con población general (Wiecko, 2010), con características homogéneas y cuyo recuerdo de aquellos acontecimientos experimentados en edades más tempranas permanece con mayor facilidad (Henry, Moffitt, Caspi, Langley y Silva, 1994).

De hecho, la prevalencia de acontecimientos traumáticos en estudiantes parece ser similar, o incluso en algunos casos mayor, a la encontrada en población general (Watson y Haynes, 2007). Por ejemplo, en los Estados Unidos algunos autores destacan un elevado porcentaje de acontecimientos potencialmente traumáticos en universitarios (oscilando entre un $52 \%$ y un $94 \%$ ) (Bernat, Ronfeldt, Calhoun y Arias, 1998; Briere, Kaltman y Green, 2008; Elhai y Simons, 2007; Frazier et al., 2009; Lauterbach y Vrana, 2001; Owens y Chard, 2006; Vrana y Lauterbach, 1994; Watson y Haynes, 2007), con una media que se sitúa cercana a la vivencia de tres acontecimientos traumáticos a lo largo de la vida del individuo (Frazier et al., 2009; Vrana y Lauterbach, 1994).

Cabe añadir, que son diversos los estudios que muestran que varones y mujeres difieren en el tipo de acontecimientos traumáticos que experimentan. En general, son los varones los que tienden a reportar acontecimientos principalmente relacionados con accidentes y situaciones de riesgo vital (i.e. agresiones, atracos, accidentes graves, guerra), mientras que las mujeres manifiestan ciertas formas de violencia más vinculadas a la agresión sexual o la violencia de pareja (Bernat et al., 1998; Breslau et al., 1998; Frazier et al., 2009; Kessler et al., 1995; Vrana y Lauterbach, 1994).

Desde una perspectiva cross-cultural, se ha confirmado la elevada prevalencia de acontecimientos traumáticos y su diferente tipología en varones y mujeres, obteniéndose resultados similares a los encontrados con muestras norteamericanas en universitarios de Israel (Amir y Sol, 1999), Turquía (Aydin, Altindag y Ozkan, 2009), Alemania, India y SudÁfrica (McAndrew, Akande, Turner y Sharma, 1998), España y Latinoamérica (Bados, Toribio y García-Grau, 2008; Martín y de Paúl, 2005a, 2005b; Vázquez, Panadero, y Rincón, 2006). A pesar de estas similitudes, el hecho que en la mayoría de estudios las muestras sean norteamericanas no permite describir un perfil completo de la exposición al trauma en diferentes culturas (Bedard, Greif y Buckley, 2004).

La frecuencia de acontecimientos traumáticos en población general y universitaria, no clínica, parece contradecir la idea defendida por estudios pioneros como el de Tennant y Andrews (1978), quienes sugerían que el desarrollo de trastornos psiquiátricos se encontraba tan determinado por la vivencia de acontecimientos traumáticos, como la vivencia de este tipo de acontecimientos por la presencia de trastornos psiquiátricos. Los estudios realizados hasta el momento constatan que incluso en poblaciones no psiquiátricas, la vivencia de acontecimientos traumáticos es elevada.

En esta línea, si bien se han realizado diversos estudios sobre la prevalencia de acontecimientos traumáticos en jóve- nes de otros países, los estudios nacionales llevados a cabo en España se han centrado, primordialmente, en la prevalencia de trastorno por estrés postraumático u otras consecuencias psicopatológicas (Bados et al., 2008; Martín y de Paúl, 2005a), así como en la exposición a determinadas experiencias traumáticas como el abuso sexual infantil (Pereda y Forns, 2007). Son dos los trabajos publicados en nuestro país centrados, específicamente, en la prevalencia de acontecimientos traumáticos en universitarios españoles (Bados, Greco y Toribio, 2012; Martín y de Paúl, 2005b), siendo de gran importancia aportar datos adicionales a partir de una muestra de similares características.

Así pues, el presente trabajo tiene como objetivo describir la prevalencia de aquellos acontecimientos potencialmente traumáticos experimentados por un amplio grupo de universitarios e identificar qué eventos son percibidos como los causantes de mayor malestar. Se pretende también comparar los resultados en función del sexo del estudiante, dadas las diferencias encontradas por estudios anteriores respecto a esta variable (Bernat et al., 1998; Breslau et al., 1998; Frazier et al., 2009; Kessler et al., 1995; Martín y de Paul, 2005b; Vrana y Lauterbach, 1994).

\section{Método}

\section{Participantes}

La muestra se compone de 1033 estudiantes universitarios de entre 18.0 y 30.6 años $(M=21.71 ; D T=2.63)$, pertenecientes a distintas facultades y centros adscritos de la Universidad de Barcelona con 317 varones (30.7\%), cuya media de edad es de 21.9 años $(D T=2.67)$, y 716 mujeres $(69.3 \%)$, cuya media de edad es de 21.6 años $(D T=2.61)$. La selección de la muestra siguió criterios de proporcionalidad en función del número de estudiantes matriculados en las distintas Divisiones Académicas y centros adscritos de la Universidad de Barcelona, situándose la proporcionalidad de la muestra en un valor promedio de $1.75 \%$. El elevado porcentaje de mujeres es similar a la distribución por sexos de los estudiantes de la Universidad de Barcelona en el curso $2001 / 2002$ (63.10\% mujeres).

\section{Instrumentos}

La identificación de acontecimientos potencialmente traumáticos se realizó a partir del cuestionario Traumatic Life Events Questionnaire (TLEQ) (Kubany, 2004; Kubany y Haynes, 2001). El instrumento evalúa la vivencia de 22 acontecimientos vitales potencialmente traumáticos, siguiendo la definición del DSM-IV-TR para acontecimientos traumáticos (APA, 2002), pero incluye un espectro de sucesos más amplio que los restringidos al criterio A1 (como el acoso sexual o sufrir una enfermedad crónica), puesto que también se han relacionado con la presencia de sintomatología postraumática (Long et al., 2008). A su vez, y en la línea de los estudios iniciados por Lazarus y Folkman (1984) quienes defienden la importancia que tiene la evaluación que haga el individuo del 
estresor como amenazante y desbordante de sus recursos en su posible posterior efecto negativo en el estado psicológico, el cuestionario incluye un ítem en el que el estudiante debe elegir aquél acontecimiento causante de un mayor malestar. El orden de presentación de los acontecimientos en el cuestionario sigue una graduación, definida por los autores originales del cuestionario, desde aquellos acontecimientos externos al individuo (por ejemplo, desastres naturales, accidentes de tráfico) a aquellos más personales y referidos, principalmente, a violencia interpersonal (por ejemplo, ser testigo de violencia doméstica o ser víctima de castigos físicos graves durante la infancia), lo que permite reducir el posible rechazo de los encuestados a aquellas preguntas más intrusivas, cuando éstas son presentadas al inicio del cuestionario. Cuando la respuesta a la vivencia del acontecimiento es positiva, el sujeto debe responder a un conjunto de cuestiones de profundización sobre el suceso referidas al número de veces que se produjo (en una categorización de nunca a más de cinco veces), las heridas sufridas como consecuencia del mismo, y la vivencia de sentimientos de miedo, terror o indefensión (criterio A2) (DSM-IV-TR; APA, 2002). Los estudios originales (Kubany y Haynes, 2001) y la adaptación española (Pereda, 2006), muestran adecuadas propiedades psicométricas del cuestionario.

\section{Procedimiento}

Una vez seleccionados aleatoriamente los centros que iban a participar, se estableció contacto con los respectivos jefes de estudio. Si se denegaba la petición de colaboración $(3.8 \%)$ o no se obtenía respuesta $(38.5 \%)$, se elegía, de forma aleatoria, otro centro del mismo ámbito de conocimiento. Las aulas se seleccionaban, a su vez, aleatoriamente en función de las posibilidades del centro, según los criterios de proporcionalidad establecidos. El día de la aplicación se realizó una breve presentación a los estudiantes en la que se solicitaba su colaboración para responder a una serie de cuestiones, requiriéndoles máxima sinceridad, y asegurándoles la confidencialidad de los datos aportados. Todos los estudiantes colaboraron y ninguno de ellos interrumpió la aplicación abandonando la sala. Tres investigadoras licenciadas en psicología acudían a las sesiones de recogida de datos, facilitando la aplicación y atendiendo a las posibles dudas planteadas por los estudiantes. Tras la respuesta de los estudiantes al cuestionario se les ofrecía un teléfono de atención personal. Únicamente dos estudiantes solicitaron este servicio. Tras finalizar el estudio se entregó un resumen global con los resultados más importantes de cada facultad y centro adscrito a sus respectivos jefes de estudio.

\section{Análisis de datos}

Para el análisis de la prevalencia de acontecimientos potencialmente traumáticos se han obtenido los porcentajes de citación de cada uno de ellos, para el total de la muestra y según el sexo de los participantes, independientemente de la valoración del sujeto de acuerdo al criterio A2 (sentimientos de miedo intenso, indefensión o terror durante el acontecimiento) (DSM-IV-TR; APA, 2002). También se han calculado las diferencias de porcentajes en función del sexo. Asimismo, se ha analizado la recurrencia en los participantes de un mismo acontecimiento, teniendo en cuenta el número de estudiantes que manifiestan haber experimentado el acontecimiento el máximo de ocasiones que permite el cuestionario (más de cinco veces). Finalmente, se ha analizado qué acontecimientos son valorados como causantes de un mayor malestar por el estudiante, y se ha calculado la ratio entre la frecuencia con que el acontecimiento ha sido considerado como el causante de mayor malestar en relación a la frecuencia de ocurrencia de tal acontecimiento. Para reducir la probabilidad de cometer errores tipo I, debido al elevado número de comparaciones, se ha utilizado la corrección de Bonferroni en los diferentes análisis realizados (Balluerka y Bergara, 2002).

\section{Resultados}

\section{Prevalencia de acontecimientos potencialmente traumáticos}

El $92.3 \%$ de los estudiantes $(93.4 \%$ de los varones y $91.8 \%$ de las mujeres) manifestó haber experimentado a lo largo de su vida al menos un acontecimiento potencialmente traumático, de los evaluados en el cuestionario. La media de acontecimientos distintos vivenciados por los estudiantes fue de $3.58(D T=2.47)$. Se observaron diferencias significativas en el número de acontecimientos experimentados por varones $(M=3.86 ; D T=2.60)$ y mujeres $(M=3.45 ; D T=2.40)$, $(t(1031)=2.48 ; p=.013 ; d=.167)$, siendo los varones aquellos que presentaron un mayor número de acontecimientos, si bien esta diferencia es trivial. La Figura 1 muestra el porcentaje de estudiantes según el número de acontecimientos vivenciados (rango de cero a 14). Puede observarse que el $73 \%$ de los estudiantes ha experimentado entre uno y cinco acontecimientos mientras que la vivencia de seis a 14 eventos ocurre en cerca del $20 \%$ de éstos.

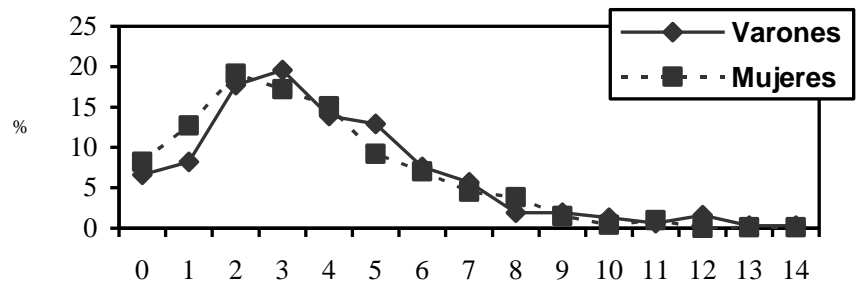

Número de acontecimientos potencialmente traumáticos

Figura 1. Porcentajes de estudiantes varones y mujeres, según el número de acontecimientos potencialmente traumáticos reportados.

Los acontecimientos experimentados más frecuentemente fueron la muerte repentina e inesperada de un ser querido $(64.5 \%)$ y la enfermedad, agresión o accidente grave de un ser querido (37.5\%) (ver Tabla 1). El resto de situaciones ob- 
tuvo porcentajes de ocurrencia inferiores al 30\%; cinco muestran una ocurrencia situada entre el 20 y el $29 \%$, ocho se producen en un porcentaje que alcanza entre el 10\% y el $19.5 \%$, y ocho más se presentan con una ocurrencia de entre el $0 \%$ y el $9 \%$.

La comparación entre varones y mujeres, aplicando un nivel de significación de $p=.002$ según la corrección de
Bonferroni, pone de manifiesto que los hombres experimentan más frecuentemente accidentes de tráfico, atracos, agresiones físicas o amenazas graves o que son testigos de agresiones con más frecuencia. Las mujeres señalan más frecuentemente haber experimentado acoso sexual y ser acechada, controlada o seguida.

Tabla 1. Acontecimientos potencialmente traumáticos, según el sexo de los estudiantes.

\begin{tabular}{|c|c|c|c|c|c|}
\hline Ítem & $\begin{array}{l}\text { Varones } \\
(n=317)\end{array}$ & $\begin{array}{l}\text { Mujeres } \\
(n=716)\end{array}$ & $\begin{array}{l}\text { Total } \\
(n=1033)\end{array}$ & z & $p$ \\
\hline 1. Desastre natural & $59(18.6 \%)$ & $119(16.6 \%)$ & $178(17.2 \%)$ & 0.78 & n.s. \\
\hline 2. Accidente de tráfico grave & $90(28.4 \%)$ & $139(19.4 \%)$ & $229(22.2 \%)$ & 3.20 & $<.001$ \\
\hline 3. Accidente grave (no de tráfico) & $75(23.6 \%)$ & $136(19.0 \%)$ & $211(20.4 \%)$ & 1.71 & .04 \\
\hline 4. Vivir, trabajar o realizar servicio en zona en guerra & $1(0.3 \%)$ & $1(0.1 \%)$ & $2(0.2 \%)$ & 0.59 & n.s. \\
\hline 5. Muerte repentina e inesperada de un ser querido & $208(65.6 \%)$ & $458(64.0 \%)$ & $666(64.5 \%)$ & 0.51 & n.s. \\
\hline 6. Enfermedad, agresión o accidente grave en un ser querido & $103(32.5 \%)$ & $283(39.5 \%)$ & $386(37.4 \%)$ & 2.15 & .02 \\
\hline 7. Sufrir una enfermedad grave & $33(10.4 \%)$ & $72(10.0 \%)$ & $105(10.2 \%)$ & 0.17 & n.s. \\
\hline 8. Atracado o testigo de un atraco con arma & $97(30.6 \%)$ & $104(14.5 \%)$ & $201(19.5 \%)$ & 6.02 & $<.001$ \\
\hline 9. Agresión física grave por un desconocido & $93(29.3 \%)$ & $82(11.4 \%)$ & $175(16.9 \%)$ & 7.07 & $<.001$ \\
\hline 10. Testigo de una agresión física grave & $100(31.5 \%)$ & $165(23.0 \%)$ & $265(25.7 \%)$ & 2.88 & .002 \\
\hline 11. Amenazas graves & $108(34.1 \%)$ & $100(14.0 \%)$ & $208(20.1 \%)$ & 7.43 & $<.001$ \\
\hline 12. Maltrato físico durante la infancia & $12(3.8 \%)$ & $38(5.3 \%)$ & $50(4.8 \%)$ & 1.05 & n.s. \\
\hline 13. Testigo de violencia familiar & $39(12.3 \%)$ & $84(11.7 \%)$ & $123(11.9 \%)$ & 0.26 & n.s. \\
\hline 14. Agresión física por parte de la pareja & $25(7.9 \%)$ & $34(4.7 \%)$ & $59(5.7 \%)$ & 2.00 & .02 \\
\hline 15. Abuso sexual antes de los 13 años por alguien al menos cinco años mayor & $25(7.9 \%)$ & $93(13.0 \%)$ & $118(11.4 \%)$ & 2.38 & .01 \\
\hline 16. Abuso sexual antes de los 13 años por alguien de similar edad & $17(5.4 \%)$ & $43(6.0 \%)$ & $60(5.8 \%)$ & 0.41 & n.s. \\
\hline 17. Abuso sexual entre los 13 y los 18 años & $14(4.4 \%)$ & $41(5.7 \%)$ & $55(5.3 \%)$ & 0.86 & n.s. \\
\hline 18. Abuso sexual después de los 18 años & $7(2.2 \%)$ & $29(4.0 \%)$ & $36(3.5 \%)$ & 1.49 & n.s. \\
\hline 19. Acoso sexual & $15(4.7 \%)$ & $106(14.8 \%)$ & $121(11.7 \%)$ & 4.64 & $<.001$ \\
\hline 20. Ser acechado/a, controlado/a, seguido/a & $40(12.6 \%)$ & $180(25.1 \%)$ & $220(21.3 \%)$ & 4.53 & $<.001$ \\
\hline 21. Aborto espontáneo & $5(1.6 \%)$ & $6(0.8 \%)$ & $11(1.1 \%)$ & 1.07 & n.s. \\
\hline 22. Aborto provocado & $12(3.8 \%)$ & $32(4.5 \%)$ & $44(4.3 \%)$ & 0.50 & n.s. \\
\hline 23. Otro acontecimiento & $48(15.1 \%)$ & $124(17.3 \%)$ & $172(16.6 \%)$ & 0.87 & n.s. \\
\hline Ninguno & $21(6.6 \%)$ & $59(8.2 \%)$ & $80(7.7 \%)$ & 0.90 & n.s. \\
\hline
\end{tabular}

n.s.: no significativo

Nota: Puesto que en diversas ocasiones un mismo estudiante pudo añadir más de un acontecimiento potencialmente traumático, la suma de los porcentajes puede ser mayor a 100 .

\section{Recurrencia de los acontecimientos reportados}

En segundo lugar, con el fin de identificar qué eventos presentan una recurrencia más elevada se ha analizado cuáles de ellos aglutinan un mayor porcentaje de respuestas en la categoría "más de cinco veces". Los porcentajes que se comentan a continuación han sido calculados a partir del número total de casos positivos (una o más veces) y no en relación al número total de participantes.

Así, los acontecimientos que presentan mayor recurrencia hacen referencia, en todos los casos, a situaciones de violencia interpersonal. Ser testigo de violencia familiar es el acontecimiento más recurrente, el $46.5 \%$ informa haberlo sufrido más de cinco veces $(48.7 \%$ en los varones y $45.2 \%$ en las mujeres); seguido de los contactos sexuales no deseados antes de los 13 años por alguien al menos cinco año mayor, que ocurre en el $37.3 \%$ más de cinco veces $(40.0 \%$ en los varones y $36.6 \%$ en las mujeres). Otros dos eventos de elevada recurrencia son el haber sido víctima de castigos físicos graves durante la infancia $(36.0 \%)$ y haber tenido contactos sexuales entre los 13 y los 18 años sin consentimiento por alguien de similar edad (30.9\%). Si bien estos cuatro acontecimientos no son aquellos más frecuentes entre los estudiantes de la muestra, sí son los que presentan una repetición de ocurrencia mayor. El resto de acontecimientos muestran niveles de recurrencia inferiores al 30\% para la respuesta de "más de cinco veces".

\section{Otros acontecimientos traumáticos}

En el listado de acontecimientos potencialmente traumáticos se delimita a 22 el número de sucesos a valorar. No obstante, dado que la conceptualización de un acontecimiento como traumático depende de la valoración que de él hacen los sujetos, el cuestionario incorpora un ítem en el que el estudiante puede indicar otros acontecimientos de similares características traumáticas que haya experimentado. Un total de 172 sujetos, el $6.6 \%$ del total (48 varones y 124 mujeres), han indicado la presencia de "otros acontecimientos". En la Tabla 2 se presentan los seis acontecimientos que han sido indicados como más frecuentes por los estudiantes. 
Tabla 2: Categoría "Otro acontecimiento" (ítem 23) según el sexo de los estudiantes.

\begin{tabular}{llllll}
$\begin{array}{l}\text { Item "Otro } \\
\text { acontecimiento" }\end{array}$ & $\begin{array}{l}\text { Varones } \\
(n=317)\end{array}$ & $\begin{array}{l}\text { Mujeres } \\
(n=716)\end{array}$ & $\begin{array}{l}\text { Total } \\
(n=1033)\end{array}$ & z & $p$ \\
\hline 1. Perderse & 14 & 20 & 34 & 1.93 & .03 \\
& $(4.4 \%)$ & $(2.8 \%)$ & $(3.3 \%)$ & & \\
2. Ver persona & 9 & 22 & 31 & 0.15 & n.s. \\
muerta & $(2.8 \%)$ & $(3.1 \%)$ & $(3.0 \%)$ & & \\
3. Muerte de un & 6 & 23 & 29 & 0.95 & n.s \\
animal doméstico & $(1.9 \%)$ & $(3.2 \%)$ & $(2.8 \%)$ & & \\
4. Sufrir el ataque de & 3 & 14 & 17 & 0.99 & n.s \\
un animal & $(0.9 \%)$ & $(1.9 \%)$ & $(1.6 \%)$ & & \\
5. Testigo de un & 4 & 10 & 14 & 0.06 & n.s \\
accidente grave & $(1.3 \%)$ & $(1.4 \%)$ & $(1.3 \%)$ & & \\
6. Ruptura relación & 5 & 2 & 7 & 2.62 & .004 \\
sentimental & $(1.6 \%)$ & $(0.3 \%)$ & $(0.7 \%)$ & & \\
\hline m.s. no significativo & & & & &
\end{tabular}

n.s.: no significativo

Perderse, ver a una persona muerta, experimentar la muerte de un animal doméstico, sufrir el ataque de un animal, ser testigo de un accidente grave y la ruptura de una relación sentimental, son aquellos acontecimientos no incluidos en el cuestionario que los estudiantes añaden más frecuentemente. Aplicando la corrección de Bonferroni $(p<$ $.008)$, se ha obtenido una única diferencia significativa según el sexo que corresponde a la vivencia de la ruptura de relaciones sentimentales, en la que los varones añaden esta situación a las mencionadas en el test con más frecuencia que las mujeres. Sin embargo, debe tenerse en cuenta que la baja ocurrencia de este suceso aconseja relativizar este resultado.

\section{Vivencia de acontecimientos causantes de un mayor malestar}

Tras la enumeración de todos los acontecimientos vivenciados, el individuo debe elegir aquél suceso que le ha causado un mayor malestar. En la Tabla 3 se expresan las ratios entre las frecuencias de citación del acontecimiento como aquél causante de mayor malestar y la ocurrencia general del acontecimiento, por sexos y en total.

Como indican los resultados, los contactos sexuales no deseados antes de los 13 años por alguien al menos cinco años mayor pasa a ser considerado como causante de mayor malestar por el $71.2 \%$ de los sujetos que lo han vivenciado. Esta ratio es significativamente superior para los varones $(92 \%)$ que para las mujeres $(65.6 \%)$. En segundo lugar, se situaría la muerte de un ser querido, considerado cómo aquel acontecimiento causante de mayor malestar por el $55.7 \%$ de los sujetos que lo han vivenciado. Destaca también el vivir, trabajar o realizar servicio en una zona en guerra como causante del mayor malestar en un porcentaje amplio de los sujetos $(50 \%)$, pero este resultado debe relativizarse debido a su escasa ocurrencia $(0.2 \%)$.

Asimismo, destacan un conjunto de acontecimientos de baja ocurrencia general (inferior a un 10\%) que son considerados como los causantes de mayor malestar por entre el $30 \%$ y el $40 \%$ de los sujetos que los han experimentado. Es- tos acontecimientos se refieren a castigos físicos graves durante la infancia $(36.0 \%)$, que son vividos como causantes de

Tabla 3. Ratios entre la frecuencia de acontecimientos causantes de un mayor malestar y la frecuencia de acontecimientos experimentados por los estudiantes, según el sexo de los estudiantes.

\begin{tabular}{|c|c|c|c|c|c|}
\hline Item & $\begin{array}{l}\text { Varones } \\
(n=317)\end{array}$ & $\begin{array}{l}\text { Mujeres } \\
(n=716)\end{array}$ & $\begin{array}{l}\text { Total } \\
(n=1033)\end{array}$ & $z$ & $p$ \\
\hline Desastr & $\begin{array}{l}5 / 59 \\
(8.4 \%)\end{array}$ & $\begin{array}{l}8 / 119 \\
(7.6 \%)\end{array}$ & $\begin{array}{l}13 / 178 \\
(7.3 \%)\end{array}$ & 0.42 & \\
\hline $\begin{array}{l}\text { Accidente de tráfico gra- } \\
\text { re }\end{array}$ & $\begin{array}{l}24 / 90 \\
(26.6 \%)\end{array}$ & $\begin{array}{l}36 / 139 \\
(25.9 \%)\end{array}$ & $\begin{array}{l}60 / 229 \\
(26.2 \%)\end{array}$ & 0.13 & 11 \\
\hline $\begin{array}{l}\text { 3. Accidente grave (no de } \\
\text { tráfico) }\end{array}$ & $\begin{array}{l}9 / 75 \\
(12.0 \%)\end{array}$ & $\begin{array}{l}9 / 136 \\
(6.6 \%)\end{array}$ & $\begin{array}{l}18 / 211 \\
(8.5 \%)\end{array}$ & 1.34 & $\mathrm{n}$ \\
\hline $\begin{array}{l}\text { 4. Vivir, trabajar o realizar } \\
\text { servicio en zona en guerra }\end{array}$ & - & $\begin{array}{l}1 / 1 \\
(100 \%)\end{array}$ & $\begin{array}{l}1 / 2 \\
(50 \%)\end{array}$ & - & \\
\hline $\begin{array}{l}\text { 5. Muerte repentina e ines- } \\
\text { perada de un ser querido }\end{array}$ & $\begin{array}{l}123 / 208 \\
(59.1 \%)\end{array}$ & $\begin{array}{l}248 / 458 \\
(54.2 \%)\end{array}$ & $\begin{array}{l}371 / 666 \\
(55.7 \%)\end{array}$ & 1.20 & $\mathrm{n}$ \\
\hline $\begin{array}{l}\text { 6. Enfermedad, agresión o } \\
\text { accidente grave en un ser } \\
\text { querido }\end{array}$ & $\begin{array}{l}14 / 103 \\
(13.6 \%)\end{array}$ & $\begin{array}{l}69 / 283 \\
(24.4 \%)\end{array}$ & $\begin{array}{l}83 / 386 \\
(21.5 \%)\end{array}$ & 2.28 & .0 \\
\hline $\begin{array}{l}\text { 7. Sufrir una enfermedad } \\
\text { grave }\end{array}$ & $\begin{array}{l}5 / 33 \\
(15.1 \%)\end{array}$ & $\begin{array}{l}9 / 72 \\
(12.5 \%)\end{array}$ & $\begin{array}{l}14 / 105 \\
(13.3 \%)\end{array}$ & 0.37 & $\mathrm{n}$ \\
\hline $\begin{array}{l}\text { 8. Atracado o testigo de un } \\
\text { atraco con arma }\end{array}$ & $\begin{array}{l}19 / 97 \\
(19.6 \%)\end{array}$ & $\begin{array}{l}14 / 104 \\
(13.5 \%)\end{array}$ & $\begin{array}{l}33 / 201 \\
(16.4 \%)\end{array}$ & 1.17 & $\mathrm{n}$ \\
\hline $\begin{array}{l}\text { física grave por } \\
\text { locido }\end{array}$ & $\begin{array}{l}15 / 93 \\
(16.1 \%)\end{array}$ & $\begin{array}{l}7 / 82 \\
(8.5 \%)\end{array}$ & $\begin{array}{l}22 / 175 \\
(12.6 \%)\end{array}$ & 1.51 & $\mathrm{n}$ \\
\hline $\begin{array}{l}\text { 10. Testigo de una agresión } \\
\text { física grave }\end{array}$ & $\begin{array}{l}5 / 100 \\
(5.0 \%)\end{array}$ & $\begin{array}{l}13 / 165 \\
(7.8 \%)\end{array}$ & $\begin{array}{l}18 / 265 \\
(6.8 \%)\end{array}$ & 0.93 & $\mathrm{n}$ \\
\hline 11. Am & $\begin{array}{l}7 / 108 \\
(6.5 \%)\end{array}$ & $\begin{array}{l}7 / 100 \\
(7.0 \%)\end{array}$ & $\begin{array}{l}14 / 208 \\
(6.7 \%)\end{array}$ & 0.14 & $\mathrm{n}$ \\
\hline $\begin{array}{l}\text { 12. Maltrato físico durante } \\
\text { la infancia }\end{array}$ & $\begin{array}{l}1 / 12 \\
(8.3 \%)\end{array}$ & $\begin{array}{l}17 / 38 \\
(44.7 \%)\end{array}$ & $\begin{array}{l}18 / 50 \\
(36.0 \%)\end{array}$ & 2.29 & .0 \\
\hline $\begin{array}{l}\text { 13. Testigo de violencia fa- } \\
\text { miliar }\end{array}$ & $\begin{array}{l}9 / 39 \\
(23.0 \%)\end{array}$ & $\begin{array}{l}29 / 84 \\
(34.5 \%)\end{array}$ & $\begin{array}{l}38 / 123 \\
(30.9 \%)\end{array}$ & 1.28 & $\mathrm{n}$ \\
\hline $\begin{array}{l}\text { 14. Agresión física por parte } \\
\text { de la pareja }\end{array}$ & $\begin{array}{l}1 / 25 \\
(4.0 \%)\end{array}$ & $\begin{array}{l}4 / 34 \\
(11.8 \%)\end{array}$ & $\begin{array}{l}5 / 59 \\
(8.5 \%)\end{array}$ & 1.05 & $\mathrm{n}$ \\
\hline 15. Abuso sexual antes de & $23 / 25$ & $61 / 93$ & $84 / 118$ & 2.58 & .00 \\
\hline $\begin{array}{l}\text { los } 13 \text { años por alguien al } \\
\text { menos cinco años mayor }\end{array}$ & $(92.0 \%)$ & $(65.6 \%)$ & $(71$. & & \\
\hline $\begin{array}{l}\text { 16. Abuso sexual antes de } \\
\text { los } 13 \text { años por alguien de } \\
\text { similar edad }\end{array}$ & $\begin{array}{l}9 / 17 \\
(52.9 \%)\end{array}$ & $\begin{array}{l}17 / 43 \\
(39.5 \%)\end{array}$ & $\begin{array}{l}26 / 60 \\
(43.3 \%)\end{array}$ & 0.94 & $\mathrm{n}$ \\
\hline $\begin{array}{l}\text { 17. Abuso sexual entre los } \\
13 \text { y los } 18 \text { años }\end{array}$ & $\begin{array}{l}6 / 14 \\
(42.8 \%)\end{array}$ & $\begin{array}{l}13 / 41 \\
(31.7 \%)\end{array}$ & $\begin{array}{l}19 / 55 \\
(34.5 \%)\end{array}$ & 0.76 & $\mathrm{n}$ \\
\hline $\begin{array}{l}\text { 18. Abuso sexual después } \\
\text { de los } 18 \text { años }\end{array}$ & $\begin{array}{l}3 / 7 \\
(42.8 \%)\end{array}$ & $\begin{array}{l}12 / 29 \\
(41.4 \%)\end{array}$ & $\begin{array}{l}15 / 36 \\
(41.6 \%)\end{array}$ & 0.07 & $\mathrm{n}$ \\
\hline 19. Ac & $\begin{array}{l}2 / 15 \\
(13.3 \%)\end{array}$ & $\begin{array}{l}16 / 106 \\
(15.9 \%)\end{array}$ & $\begin{array}{l}18 / 121 \\
(14.9 \%)\end{array}$ & 0.18 & $\mathrm{n}$ \\
\hline $\begin{array}{l}\text { 20. Ser acechado/a, } \\
\text { controlado/a, seguido/a }\end{array}$ & - & $\begin{array}{l}18 / 180 \\
(1.0 \%)\end{array}$ & $\begin{array}{l}18 / 220 \\
(8.2 \%)\end{array}$ & - & \\
\hline 21. Aborto espontáneo & $\begin{array}{l}2 / 5 \\
(40.0 \%)\end{array}$ & $\begin{array}{l}1 / 6 \\
(16.6 \%)\end{array}$ & $\begin{array}{l}3 / 11 \\
(27.3 \%)\end{array}$ & 0.86 & $\mathrm{n}$ \\
\hline 22. Aborto provocado & $\begin{array}{l}5 / 12 \\
(41.6 \%)\end{array}$ & $\begin{array}{l}12 / 32 \\
(37.5 \%)\end{array}$ & $\begin{array}{l}17 / 44 \\
(38.6 \%)\end{array}$ & 0.25 & $\mathrm{n}$ \\
\hline 23. Otro a & $\begin{array}{l}9 / 48 \\
(18.7 \%)\end{array}$ & $\begin{array}{l}36 / 124 \\
(29.0 \%)\end{array}$ & $\begin{array}{l}45 / 172 \\
(26.1 \%) \\
\end{array}$ & 1.37 & n. \\
\hline
\end{tabular}

n.s.: no significativo

gran malestar especialmente por las mujeres (44.7\%), a contactos sexuales no deseados en sus distintas formas (antes de los 13 años por alguien de similar edad (43.3\%), entre los 13 y los 18 años $(34.5 \%$ ) y después de los 18 años $(41.6 \%)$ y al aborto provocado $(38.6 \%)$. El aborto espontáneo, que es 
considerado un acontecimiento potencialmente traumático por un $1.1 \%$ de la población, es indicado como causante de mayor malestar en un $40 \%$ de los varones.

Tomando en consideración el suceso más frecuentemente identificado como el causante de mayor malestar, concretamente los contactos sexuales no deseados antes de los 13 años por alguien al menos cinco años mayor, se ha considerado de interés comparar aquellos estudiantes que han vivido este acontecimiento pero que no lo seleccionan como el causante de mayor malestar y aquellos que sí lo han seleccionado.

El número de acontecimientos experimentados por el grupo que no selecciona este evento como el de mayor malestar es significativamente superior $(M=6.31 ; D T=2.33)$ al de aquellos que sí lo eligen $(M=4.57 ; D T=2.88, t(116)=$ $3.19 ; p=.002 ; d=0.64)$. Por otro lado, la frecuencia del abuso suele ser menor en aquellos que no lo eligen (rango de $1 \mathrm{a}$ $6, M=2.09 ; D T=2.02)$ de la de los que sí lo eligen $(M=$ $3.47 ; D T=2.39, t(116)=-3.22 ; p=.002 ; d=.65)$. Cabe añadir que el grupo que no selecciona los contactos sexuales no deseados como evento de mayor malestar tiende a elegir en su lugar la muerte repentina e inesperada de un ser querido (en un $28.6 \%$ de los casos) y ser testigo de violencia familiar (en el 14.3\%).

\section{Discusión y conclusiones}

Los resultados obtenidos en este trabajo aportan información sobre el frecuentemente no detectado historial traumático de la población universitaria (Elhai y Simons, 2007), con una prevalencia muy elevada de sucesos experimentados (93.4\% varones y $91.8 \%$ mujeres), similar a la encontrada por Martín y De Paúl (2005b), pero que supera los porcentajes encontrados en otros estudios nacionales (Bados et al., 2008; Bados et al., 2012) e internacionales (Amir y Sol, 1999; Aydin et al., 2009; Bernat et al., 1998; Briere et al., 2008).

La consideración de unos u otros acontecimientos como potencialmente traumáticos, así como la forma en que éstos se evalúan en cada estudio puede haber influido en las diferentes tasas de prevalencia encontradas en las distintas publicaciones. El presente trabajo incluye una definición amplia de acontecimientos potencialmente traumáticos y obtiene una alta prevalencia en la línea de los trabajos de Vrana y Lauterbach (1994), Watson y Haynes (2007) y Frazier et al. (2009) con metodologías similares. Cabe destacar que la valoración de qué acontecimientos pueden ser considerados traumáticos es un tema controvertido que sigue discutiéndose en la actualidad (Brewin, 2008).

Según los datos de este trabajo, los estudiantes varones indican haber experimentado un número ligeramente superior de este tipo de acontecimientos respecto a las mujeres. Esta diferencia requiere de un análisis más exhaustivo ya que ha sido constatada en una parte de los estudios publicados al respecto, tanto en población general (Breslau et al., 1998; Darves-Bornoz et al., 2008; Kessler et al., 1995; Stein et al., 1997) como en estudiantes universitarios (Amir y Sol, 1999;
Vrana y Lauterbach, 1994), pero no se ha detectado en otros trabajos nacionales (Martín y de Paúl, 2005b) o incluso se han obtenido resultados en una línea opuesta (Frazier et al., 2009).

A excepción de la muerte repentina e inesperada de un ser querido, que es el acontecimiento más frecuentemente experimentado independientemente del sexo del estudiante, tal como indican estudios previos realizados en España (Bados et al., 2012; Martín y de Paúl, 2005b) y en otros países (población general: Breslau et al., 1998; Robin et al., 1997; Stein et al., 1997; y estudiantes universitarios: Frazier et al., 2009; Vrana y Lauterbach, 1994; Watson y Haynes, 2007), se ha detectado que varones y mujeres difieren en el tipo concreto de acontecimientos experimentados, también en la línea de lo obtenido en otros trabajos (en población general: Breslau et al., 1998; Kessler et al., 1995; Robin et al., 1997; Stein et al., 1997; y estudiantes universitarios: Amir y Sol, 1999; Bernat et al., 1998; Frazier et al., 2009; Martín y de Paúl, 2005b; Vrana y Lauterbach, 1994).

Los varones indican, de forma más frecuente, haber sido víctimas de amenazas de muerte, de agresiones físicas graves, de atracos o haber sido testigos de un atraco con arma o de una agresión física grave, y haber sufrido un accidente de tráfico grave. Por su parte, las mujeres indican más frecuentemente haber estado expuestas a acoso sexual, o haberse sentido acechadas, controladas o seguidas. Podemos interpretar que los varones indican acontecimientos que podrían definirse como de violencia explícita (amenazas, agresiones físicas), mientras que las mujeres manifiestan acontecimientos de violencia más psicológica $\mathrm{y}$, principalmente de tipo sexual (acoso, acecho). Estas diferencias en los acontecimientos que experimentan ambos sexos siguen la línea de lo obtenido en estudios epidemiológicos realizados en España (Martín y de Paúl, 2005b) y en otros países (población general: Breslau et al., 1998; y población universitaria: Bernat et al., 1998; Frazier et al., 2009; Vrana y Lauterbach, 1994), presentando importantes implicaciones clínicas, que deberían tenerse en cuenta tanto en la realización de programas de prevención primaria para jóvenes, como en los tipos de intervención a desarrollar con éstos.

Respecto al mayor porcentaje de varones que manifiestan haber sido víctimas de agresiones por parte de sus parejas, este resultado coincide con otros estudios llevados a cabo con estudiantes universitarios españoles, donde los varones afirman haber cometido más violencia contra sus parejas y, a su vez, haber sido también víctimas de más violencia ( $\mathrm{Mu}-$ ñoz-Rivas, Graña Gómez, O'Leary y González Lozano, 2007); si bien cuando se tienen en cuenta las consecuencias para la salud resultado de las agresiones, éstas suelen ser mayores para las mujeres (Straus, 1999).

Por otro lado, la menor frecuencia en la muestra de estudio del acontecimiento desastre natural (17.2\%), similar a la obtenida en otras zonas de España (Martín y de Paúl, 2005b), pero en contraposición a los resultados de otros países con población general (Kessler et al., 1995) y población universitaria (Vrana y Lauterbach, 1994), puede explicarse por las 
propias características naturales del área geográfica a la que pertenece esta muestra.

Cabe añadir que, independientemente del sexo del estudiante, algunos acontecimientos presentan como característica específica la recurrencia. Los sucesos más recurrentes hacen referencia a situaciones de violencia interpersonal vividas siempre durante la infancia o la adolescencia, como haber sido testigo de violencia doméstica, recibir castigos físicos graves y los contactos sexuales no deseados, ya sea antes de los 13 años por alguien al menos cinco años mayor o entre los 13 y los 18 años. Debe tenerse en cuenta que, si bien haber experimentado alguna forma de abuso sexual en la infancia es uno de los acontecimientos más recurrentes, en la mayoría de casos éste se produce en una única ocasión en la muestra analizada, tal y como indican otros estudios realizados con población universitaria (Edgardh y Ormstad, 2000; Goldman y Padayachi, 1997; Oaksford y Frude, 2001).

Cabe mencionar que este tipo de acontecimientos, poco frecuentes en la muestra de estudio general pero recurrentes a lo largo de la vida del estudiante, y principalmente relacionados con violencia interpersonal y agresores conocidos, son substancialmente distintos de aquellos relacionados con accidentes, enfermedades y con la violencia llevada a cabo por agresores desconocidos, mucho más frecuentes pero de escasa repetición. Sus posibles consecuencias en el estado emocional del individuo, por otro lado, también son diferentes, puesto que son los acontecimientos de violencia interpersonal, y especialmente aquellos que acontecen en la infancia, los que se relacionan con unos efectos psicológicos más graves (Finkelhor, 2007; Turner, Finkelhor y Ormrod, 2006).

Como acontecimientos potencialmente traumáticos añadidos por los estudiantes y no incluidos originalmente en el cuestionario destacan perderse, ver a una persona muerta y la muerte de un animal doméstico. Si bien se ha discutido que considerar una definición de acontecimiento traumático más amplia que la actual, que permita la inclusión de acontecimientos que no cumplen el criterio A1 (DSM-IV-TR; APA, 2002), puede conducir a un sobrediagnóstico del trastorno por estrés postraumático (Rosen, 2005), son diversos los autores que defienden que ampliar este criterio no afectaría a la conceptualización del trastorno pero sí permitiría el acceso a tratamiento a un mayor número de personas afectadas por sucesos de una gran intensidad emocional (Davidson y Foa, 1991). En este sentido, futuras adaptaciones del autoinforme original (Kubany y Haynes, 2001) deberían considerar la inclusión de algunos de estos acontecimientos aportados por el propio individuo y que son valorados por los estudiantes como generadores de un importante malestar.

La perspectiva que da énfasis a la valoración del conflicto por el propio sujeto queda realzada a partir de los datos obtenidos en este trabajo y muestra que la valoración personal que haga el individuo del acontecimiento como traumático y negativo para su bienestar debe ser tenida en cuenta por los profesionales al considerar los posibles efectos de éste (Lazarus y Folkman, 1984). Ciertamente, ofrecer la posibilidad de que el sujeto elija el acontecimiento causante de un mayor malestar según su vivencia muestra la gravedad subjetiva de ese acontecimiento, información sumamente valiosa desde una perspectiva traumatogénica. Algunos estudios realizados con poblaciones universitarias confirman este resultado (Frazier et al., 2009; Vrana y Lauterbach, 1994), si bien la mayoría de trabajos publicados únicamente evalúan la ocurrencia del acontecimiento, no la vivencia subjetiva del individuo ante éste.

El análisis de los acontecimientos vivenciados desde la perspectiva del causante de mayor malestar permite subrayar la importancia que cobra, entre otros, la experiencia de contacto sexual no deseado antes de los 13 años por alguien al menos cinco años mayor como acontecimiento traumático. De entre el porcentaje de estudiantes que han experimentado este acontecimiento (11.4\%) el $71.2 \%$ lo valoran como causante de un mayor malestar psicológico, de forma superior en los varones $(92 \%)$ que en las mujeres $(65.6 \%)$. De este modo, la experiencia de contacto sexual no deseado genera gran malestar psicológico y aparece como un acontecimiento más dañino en los varones que en las mujeres. Si bien estos datos contradicen estudios clásicos que no aportan diferencias intersexos en la vivencia de este malestar (Briere y Elliot, 1994; Finkelhor, Hotaling, Lewis y Smith, 1990), siguen la línea de otros trabajos que han mostrado que el impacto emocional del abuso sexual infantil es más fuerte en varones y puede estar relacionado con una mayor frecuencia de violencia y agresiones físicas por parte del victimario, así como por la existencia de un mayor porcentaje de agresores del mismo sexo en este grupo (Garnefski y Arends, 1998; Garnefski y Diekstra, 1997). También podría ser debido a que los varones parecen sufrir con más frecuencia episodios de abuso más invasivos que las mujeres, en las que serían más frecuentes los tocamientos (Finkelhor et al., 1990). Por otra parte, los resultados indican que el abuso sexual antes de los 13 años con asimetría de edad es raramente superado por otros acontecimientos en cuanto al malestar producido, pero que en algunos casos otros eventos pueden ocupar un lugar preponderante principalmente cuando el abuso ha sido de baja frecuencia y la persona ha experimentado gran variedad de acontecimientos, en estos casos puede tener mayor peso la muerte inesperada de un ser querido o el haber sido testigo de violencia familiar.

Cabe destacar que el aborto provocado es considerado por un $38.6 \%$ de jóvenes como el acontecimiento que genera un mayor malestar, si bien el escaso número de estudiantes que ha experimentado este acontecimiento, al igual que en otros trabajos (Frazier et al., 2009), debe tenerse en cuenta. La existencia de sintomatología postraumática tras la experiencia de un aborto ha sido constatada en estudios previos, tanto cuando éste es espontáneo (Engelhard, van den Hout y Arntz, 2001), como cuando es provocado (Bradshaw y Slade, 2003), siendo un dato que debería ser considerado por los equipos de asesoramiento y planificación familiar por sus implicaciones en la práctica profesional. Además, estos equipos deberían valorar que el efecto traumatogénico de esta experiencia es también frecuente en el hombre, por lo que es 
importante estar atentos a la reacción de ambos miembros de la pareja.

Como implicaciones para la práctica clínica y, en función de los resultados del presente trabajo, cabe sugerir que aquellos profesionales que deseen analizar el impacto de la vivencia de acontecimientos potencialmente traumáticos en el estado psicológico del individuo utilicen instrumentos que permitan una valoración personal del encuestado ante estos posibles acontecimientos, puesto que la asignación predeterminada de un determinado nivel de estrés a un acontecimiento no tiene porqué coincidir con la experiencia subjetiva del individuo evaluado.

\section{Limitaciones}

La principal limitación de este trabajo es la utilización de información retrospectiva, que siempre se encuentra determinada por la memoria del encuestado y su deseo de responder con sinceridad. La utilización de autoinformes para valorar experiencias personales como el maltrato o el abuso sexual infantil, vivencias de escasa revelación, incluso mediante instrumentos validados previamente, con adecuadas cualidades psicométricas, aumenta el riesgo de posibles falsos negativos lo que supone un importante problema en su estimación, si bien existen estudios que afirman que el uso de cuestionarios anónimos facilita enormemente el reporte de estas experiencias (Dill, Chu, Grob y Eisen, 1991). Debe tenerse en cuenta que los recuerdos sobre hechos traumáticos difieren de los recuerdos felices en algunas pocas características, quizás menos de las que podría suponerse (Shobe y Kihlstrom, 1997), si bien son más confusos y complejos, cuestan más de describir y pueden verse más deteriorados por el paso del tiempo debido al recuerdo recurrente que de estos sucesos hace la víctima (Manzanero, 2010).

Cabe considerar que la generalización de resultados se encuentra, a su vez, limitada por las propias características de la muestra universitaria, principalmente compuesta por muje-

\section{Referencias}

American Psychiatric Association. (1980). Diagnostic and statistical manual of mental disorders (3 $3^{\text {trd }}$ ed.). Washington, DC: Author.

American Psychiatric Association. (2002). Manual diagnóstico y estadístico de los trastornos mentales ( $4^{\mathrm{a}}$ ed. texto revisado). Barcelona: Masson.

Amir, M. y Sol, O. (1999). Psychological impact and prevalence of traumatic events in a student sample in Israel: The effect of multiple traumatic events and physical injury. Journal of Traumatic Stress, 12, 139-154.

Aydin, Y. E., Altindag, A. y Ozkan, M. (2009). Childhood traumatic events and dissociation in university students. International Journal of Psychiatry in Clinical Practice, 13 (1), 25-30.

Bados, A., Greco, A. y Toribio, L. (2012). Experiencias traumáticas y trastorno por estrés postraumático en universitarios españoles. Anales de Psicología, 28 (2), 387-396.

Bados, A., Toribio, L. y García-Grau, E. (2008). Traumatic events and tonic immobility. The Spanish Journal of Psychology, 11 (2), 516-521.

Báguena, M. J. (2001). Tratamientos psicológicos eficaces para el estrés post-traumático. Psicothema, 13 (3), 479-492.

Balluerka, N. y Vergara, A. I. (2002). Diseños de investigación experimental en Psicología. Madrid: Prentice-Hall.

Bedard, M., Greif, J. L. y Buckley, T. C. (2004). International publication trends in the traumatic stress literature. Journal of Traumatic Stress, 17, res, aunque ello responda a los porcentajes de inscripción del alumnado de los cursos y especialidades encuestadas. La utilización de este tipo de muestras, sin embargo, también presenta la ventaja de evitar, al menos parcialmente, las distorsiones y problemas de recuerdo que puedan presentar los adultos mayores (Henry et al., 1994) y ha sido defendida en estudios recientes (Wiecko, 2010).

Finalmente, la elevada prevalencia de acontecimientos traumáticos en la muestra analizada, superior a la encontrada en muchos de los estudios publicados previamente al respecto, puede deberse a que en el instrumento empleado se incluyen diversos acontecimientos vitales, de entre los cuales unos presentan un componente más claramente traumático que otros, según la definición establecida en el DSM-IV-TR (APA, 2002). No obstante, y en función de la propuesta de Kubany y Haynes (2001), los estudiantes informaron en un primer momento de la ocurrencia del acontecimiento independientemente del cumplimiento del criterio A2 (APA, 2002), que se refiere a la respuesta emocional subjetiva de terror, horror o indefensión por parte del individuo, y en un segundo momento seleccionaron el acontecimiento como causante de mayor malestar, lo cual informa de aquél que ha sido vivido de forma más traumática. Algunas críticas al criterio A2 (APA, 2002) y a su utilidad (Bedard-Gilligan y Zoellner, 2008) han destacado que se puede llegar al diagnóstico de trastorno por estrés postraumático (Brewin, Andrews y Rose, 2000) a partir de otro tipo de emociones distintas a las valoradas por este criterio. Ello justifica el haber tenido en cuenta la valoración subjetiva del individuo respecto al nivel de malestar asociado con el acontecimiento, más que a la vivencia específica de emociones de terror, horror o indefensión, no siempre presentes en las víctimas de acontecimientos traumáticos.

Agradecimientos.- Este trabajo ha sido parcialmente subvencionado por el Ministerio de Educación e Innovación (PSI200911542).

97-101.

Bedard-Gilligan, M. y Zoellner, L. A. (2008). The utility of the A1 and A2 criteria in the diagnosis of PTSD. Behaviour Research and Therapy, 46, 1062-1069.

Bernat, J. A., Ronfeldt, H. M., Calhoun, K. S. y Arias, I. (1998). Prevalence of traumatic events and peritraumatic predictors of posttraumatic stress symptoms in a nonclinical sample of college students. Journal of Traumatic Stress, 11, 645-664.

Bradshaw, Z. y Slade, P. (2003). The effects of included abortion on emotional experiences and relationships: A critical review of the literature. Clinical Psychology Review, 23 (7), 929-958.

Breslau, N., Kessler, R. C., Chilcoat, H. D., Schultz, L. R., Davis, G. C. y Andreski, P. (1998). Trauma and posttraumatic stress disorder in the community: The 1996 Detroit area survey of trauma. Archives of General Psychiatry, 55 (7), 626-632.

Brewin, C. (2008, Noviembre). Traumatic events should meet either Criterion A1 or A2 not both. Comunicación presentada en el 24th annual meeting de la International Society for Traumatic Stress Studies, Chicago, IL.

Brewin, C. R., Andrews, B. y Rose, S. (2000). Fear, helplessness, and horror in posttraumatic stress disorder: Investigating DSM-IV Criterion A2 in victims of violent crime. Journal of Traumatic Stress, 13 (3), 499-509. 
Briere, J. y Elliot, D. M. (1994). Immediate and long-term impacts of child sexual abuse. The Future of Children, 4 (2), 54-70.

Briere, J., Kaltman, S. y Green, B. L. (2008). Accumulated childhood trauma and symptom complexity. Journal of Traumatic Stress, 21 (2), 223226.

Darves-Bornoz, J., Alonso, J., de Girolamo, G., de Graaf, R., Haro, J., Kovess-Masfety, V., et al. (2008). Main traumatic events in Europe: PTSD in the European Study of the Epidemiology of Mental Disorders Survey. Journal of Traumatic Stress, 21, 455-462.

Davidson, J. R. T. y Foa, E. B. (1991). Diagnostic issues in posttraumatic stress disorder: considerations for the DSM-IV. Journal of Abnormal Psychology, 100, 346-355.

Dill, D. L., Chu, J. A., Grob, M. C. y Eisen, S. V. (1991). The reliability of abuse history reports: a comparison of two inquiry formats. Comprehensive Psychiatry, 32, 166-169.

Edgardh, K. y Ormstad, K. (2000). Prevalence and characteristics of sexual abuse in a national sample of Swedish seventeen-year-old boys and girls. Acta Paediatrica, 88, 310-319.

Elhai, J. D. y Simons, J. S. (2007). Trauma exposure and posttraumatic stress disorder predictors of mental health treatment use in college students. Psychological Services, 4, 38-45.

Engelhard, I. M., van den Hout, M. A. y Arntz, A. (2001). Posttraumatic stress disorder after pregnancy loss. General Hospital Psychiatry, 23 (2), 62-66.

Finkelhor, D. (2007). Developmental victimology. The comprehensive study of childhood victimizations. In R. C. Davis, A. J. Luirigio \& S. Herman (Eds.), Victims of crime (3rd ed), pp. 9-34. Thousand Oaks, CA: Sage Publications.

Finkelhor, D., Hotaling, G., Lewis, I. A. y Smith C. (1990). Sexual Abuse in a national survey of adult men and women: prevalence, characteristics, and risk factors. Child Abuse \& Neglect, 14, 19-28.

Flett, R. A., Kazantzis, N., Long, N. R., MacDonald, C. y Millar, M. (2002). Traumatic events and physical health in a New Zealand community sample. Journal of Traumatic Stress, 15 (4), 303-312.

Frazier, P., Anders, S., Perera, S., Tomich, P., Tennen, H., Park, C., et al. (2009). Traumatic events among undergraduate students: Prevalence and associated symptoms. Journal of Counseling Psychology, 56, 450-460.

Garnefski, N. y Arends, E. (1998). Sexual abuse and adolescent maladjustment: differences between male and female victims. Journal of Adolescence, $21,99-107$.

Garnefski, N. y Diekstra, R. F. W. (1997). Child sexual abuse and emotional and behavioral problems in adolescence, gender differences. Journal of the American Academy of Child and Adolescent Psychiatry, 36 (3), 323-329.

Goldman, J. D. G. y Padayachi, U. K. (1997). The prevalence and nature of child sexual abuse in Queensland, Australia. Child Abuse y Neglect, 21 (5), 489-498.

Henry, B., Moffitt, E. G., Caspi, A., Langley, J. y Silva, P. A. (1994). On the "remembrance of things past": A longitudinal evaluation of the retrospective method. Psychological Assessment, 6 (2), 92-101.

Kessler, R. C., Sonnega, A., Bromet, E., Hughes, M. y Nelson, C. B. (1995). Posttraumatic stress disorder in the National Comorbidity Survey. Archives of General Psychiatry, 52, 1048-1060.

Kubany, E. S. (2004). The Traumatic Life Events Questionnaire (TLEQ) and PTSD Screening and Diagnostic Scale (PSDS). Los Angeles, CA: Western Psychological Services.

Kubany, E. S. y Haynes, S. N. (2001). Traumatic Life Events Questionnaire. Manual. Second Draft, Western Psychological Services.

Lauterbach, D. y Vrana, S. (2001). The relationship among personality variables, exposure to traumatic events, and severity of posttraumatic stress symptoms. Journal of Traumatic Stress, 14, 29-45

Lazarus, R. S. y Folkman, S. (1984). Stress, appraisal and coping. New York: Springer Publishing Company.

Long, M. E., Elhai, J. D., Schweinle, A., Gray, M. J., Grubagh, A. L., et al. (2008). Differences in posttraumatic stress disorder diagnostic rates and symptom severity between Criterion A1 and non-Criterion A1 stressors. Journal of Anxiety Disorders, 22, 1255-1263.
Manzanero, A. (2010). Recuerdo de hechos traumáticos: De la introspección al estudio objetivo. Psicopatología Clínica, Legal y Forense, 10, 149 164.

Martín, J. L. y de Paúl, J. (2005a). Post-traumatic stress disorder in victims of traumatic events. Psychology in Spain, 9, 57-62.

Martín, J. L. y de Paúl, J. (2005b). Situaciones traumáticas: Un estudio sobre su prevalencia en población universitaria. Psicología Conductual, 13, 79-96.

McAndrew, F. T., Akande, A., Turner, S. y Sharma, Y. (1998). A crosscultural ranking of stressful life events in Germany, India, South Africa, and the United States. Journal of Cross-Cultural Psychology, 29, 717-727.

McCall, G. J. y Resick, P. A. (2003). A pilot study of PTSD symptoms among Kalahari Bushmen. Journal of Traumatic Stress, 16 (5), 445-450.

Muñoz-Rivas, M. J., Graña Gómez, J. L., O'Leary, K. y González Lozano, P. (2007). Agresión física y psicológica en las relaciones de noviazgo en universitarios españoles. Psicothema, 19 (1), 102-107.

Oaksford, K. L. y Frude, N. (2001). The prevalence and nature of child sexual abuse: evidence from a female university sample in the UK Child Abuse Review, 10, 49-59.

Organización Panamericana de la Salud, Oficina Regional para las Américas de la Organización Mundial de la Salud (2002). Informe mundial sobre la violencia y la salud: resumen. Washington, D.C.

Owens, G. P. y Chard, K. M. (2006). PTSD severity and cognitive reactions to trauma among a college sample. Journal of Aggression, Maltreatment and Trauma, 13, 23-36.

Pereda, N. (2006). Malestar psicológico en estudiantes universitarios víctimas de abuso sexual infantil y otros estresores. Tesis Doctoral no publicada, Universitat de Barcelona, Barcelona (España).

Pereda, N. y Forns, M. (2007). Prevalencia y características del abuso sexua infantil en estudiantes universitarios españoles. Child Abuse y Neglect, 31 , 417-426.

Robin, R. W., Chester, B., Rasmussen, J. K., Jaranson, J. M. y Goldman, D. (1997). Prevalence and characteristics of trauma and posttraumatic stress disorder in a southwestern American Indian community. The American Journal of Psychiatry, 154 (11), 1582-1588.

Rosen, G. M. (2005). Traumatic events, criterion creep, and the creation of posttraumatic stress disorder. The Scientific Review of Mental Health Practice, 3(2), 39-42.

Shobe, K. K. y Kihlstrom, J. F. (1997). Is traumatic memory special? Current Directions in Psychological Science, 6 (3), 70-74.

Sigales, S. R. (2006). Catástrofe, víctimas y trastornos: Hacia una definición en psicología. Anales de Psicología, 22 (1), 11-21.

Stein, M. B., Walker, J. R., Hazen, A. L. y Forde, D. R. (1997). Full and partial posttraumatic stress disorder: findings from a community survey. The American Journal of Psychiatry, 154 (8), 1114-1119.

Straus, M. (1999). The controversy over domestic violence by women. A methodological, theoretical, and sociology of science analysis. In X. B. Arriaga \& S. Oskamp (Ed.). Violence in intimate relationships (17-44). Thousand Oaks, CA: Sage.

Tennant, C. y Andrews, G. (1978). The pathogenic quality of life event stress in neurotic impairment. Archives of General Psychiatry, 35, 859-863.

Turner, H. A., Finkelhor, D. y Ormrod, R. K. (2006). The effect of lifetime victimization on the mental health of children and adolescents. Social Science \& Medicine, 62(1), 13-27.

Vázquez, J. J., Panadero, S. y Rincón, P. P. (2006). Diferencias transculturales y vivencia de sucesos estresantes entre estudiantes Latinoamericanos y españoles. Boletín de Psicología, 86, 27-51.

Vrana, S. y Lauterbach, D. (1994). Prevalence of traumatic events and posttraumatic psychological symptoms in a nonclinical sample of college students. Journal of Traumatic Stress, 7 (2), 289-302.

Watson, S. B. y Haynes, S. N. (2007). Brief screening for traumatic life events in female university health service patients. International Journal of Clinical and Health Psychology, 7, 261-282.

Wiecko, F. M. (2010). Research note: Assessing the validity of college samples: Are students really that different?. Journal of Criminal Justice, 38 , 1186-1190.

(Artículo recibido: 7-4-2011, revisado: 26-05-2011, aceptado: 19-02-2012) 